\title{
Survey of the Solvent Effect on Equilibrium Polymerization: The Case of 1,3-Dioxolan
}

\author{
Van Tam BUI and Jacques LÉONARD* \\ Department of Chemistry and Chemical Engineering, Royal Military College, \\ Kingston, Ontario, Canada, K7K $5 L 0$ \\ * CERSIM and Département de Chimie, Université Laval, \\ Québec, Québec, Canada, G1K 7 P4
}

(Received September 19, 1988)

\begin{abstract}
The use of Flory-Huggins interaction parameters to express the solvent effect on the equilibrium polymerizations is reviewed. Interactions in three binary mixtures: 1,3-dioxolan/ benzene, benzene/poly(1,3-dioxolan), and 1,3-dioxolan/poly(1,3-dioxolan) have been investigated at various temperatures using vapor pressure measurements. Calculations yield a parameter of about 0.17 for the first mixture, 0.25 for the second and 0.65 for the last one. The magnitudes of the two first parameters indicate that monomer/solvent interactions are relatively weak while solvent/ polymer interactions are rather strong so that, on the thermodynamic point of view, benzene should be regarded as a good solvent for the polymerization of 1,3-dioxolan. All those parameters were then used together with the available data of equilibrium compositions of polymerization of 1,3dioxolan to compute the solvent effect as well as the free-energy change for this reaction.

KEY WORDS Benzene / 1,3-Dioxolan / Free-Energy / Interaction Parameter / Poly(1,3-dioxolan) / Solvent Effect / Vapor Pressure /
\end{abstract}

Like any other chemical reactions carried out in a chemically inert solvent, a polymerization one may have its equilibrium state greatly affected by the intermolecular interactions between the solvent and the monomer, or the formed polymer. This nonideal behavior, in the case of small molecules reactions, is usually expressed by means of the ratio of the individual activity coefficients of the products over those of the reactants in the equilibrium constant expression. For polymerizations taking place in a solvent, if the activity coefficients are expressed by the Flory-Huggins theory, it has been shown ${ }^{1.2}$ that the effect of solvent on the whole equilibrium system can be described through the interaction parameters, $\chi$, using:

$$
\begin{aligned}
\frac{\Delta G_{\mathrm{lc}}}{R T}= & \ln \phi_{\mathrm{m}}+1+\left(\chi_{\mathrm{ms}}-\chi_{\mathrm{sp}}\left(\bar{V}_{\mathrm{m}} / \bar{V}_{\mathrm{s}}\right)\right) \phi_{\mathrm{s}} \\
& +\chi_{\mathrm{mp}}\left(\phi_{\mathrm{p}}-\phi_{\mathrm{m}}\right)
\end{aligned}
$$

where $\Delta G_{\mathrm{lc}}$ means the free-energy change upon the conversion of $1 \mathrm{~mol}$ of pure liquid monomer into 1 base-mol of amorphous polymer, $R$ is the gas constant, $T$ is the absolute temperature, $\phi$ is the volume fraction, $\bar{V}$ is the molar volume, and the subscripts $\mathrm{m}, \mathrm{s}, \mathrm{p}$, refer to monomer, solvent and polymer, respectively.

In the above expression, the whole solvent effect is represented by:

$$
\beta=\left(\chi_{\mathrm{ms}}-\chi_{\mathrm{sp}}\left(\bar{V}_{\mathrm{m}} / \bar{V}_{\mathrm{s}}\right)\right)
$$

in which the $\chi_{\mathrm{ms}}$ parameter has a predominant effect since it could largely vary from one solvent to another with respect to a given monomer while the corresponding variation of $\chi_{\text {sp }}$ parameter is usually smaller for a given polymer. This contrast may be regarded as consequences of two basically different mixing processes: between two liquid components (monomer/solvent) in one hand and between a 
liquid and a long chain polymer in the other hand. In the first case, the molecules of each liquid should prealably breakup their intermolecular forces between like molecules before establishing the intermolecular attractions with the molecules of the other liquid to become completely solvated. The enthalpy, as well as the entropy, of mixing are greatly affected by the mutual chemical natures of two liquids so that any small change in their compatibility could induce an appreciable fluctuation in the free-energy of mixing and then in the value of the interaction parameter $\chi$. On the other hand, in the second mixing process, except the cases where the intramolecular forces are very strong like with the polyamides, the solvent molecules have only to solvate around each polymer chain while the monomeric units of the latter stay linked together. Consequently, the magnitudes of the fluctuation in the enthalpy and entropy of mixing (due to the change of solvents) are smaller than those corresponding to binary liquid mixtures.

The foregoing explanations have been successfully checked by the values of $\chi_{\mathrm{ms}}$ changing from -0.30 to 0.17 and 0.55 for the $\alpha$-methylstyrene monomer when mixed with tetrahydrofuran, ${ }^{3} p$-dioxan, ${ }^{4}$ and cyclohexane, ${ }^{4}$ respectively. Meanwhile, the corresponding changes for $\chi_{\mathrm{sp}}$ parameter for poly $(\alpha$-methylstyrene) mixed in the same solvents were only from 0.42 to 0.44 and 0.51 , respectively. All those values were then successfully applied ${ }^{4}$ to eq 1 and 2 to interpret the experimental data of the equilibrium polymerization of $\alpha$-methylstyrene in the above three solvents. ${ }^{5-7}$

In the following sections, we will present first the evaluation of the interaction parameters $\chi_{\mathrm{ms}}, \chi_{\mathrm{sp}}$ and $\chi_{\mathrm{mp}}$ obtained from static vapor pressure measurements for the mixtures: 1,3-dioxolan (DOL)/benzene (BEN), $\mathrm{BEN} /$ poly(1,3-dioxolan) (PDOL) and DOL/ PDOL, at various temperatures. Then these parameters will be used along with the available data $^{8}$ of equilibrium polymerization of DOL in BEN to compute the free-energy change, $\Delta G_{\mathrm{Ic}}$, of the reaction. The solvent effect will be thereafter discussed.

\section{EXPERIMENTAL}

\section{Materials}

The monomer and solvent used (DOL and BEN) were the best available reagents. They were purified prior to their use by storing over $\mathrm{CaH}_{2}$ and degassing over a period of several days, on a vacuum manifold. They were then distilled onto a first sodium mirror and kept over a second one during the measurements.

The polymeric material used, PDOL, was prepared in our laboratory by cationic ringopening polymerization of DOL in bulk. ${ }^{9}$ Its viscometric average molecular weight, $\bar{M}_{v}$, was $9.4 \times 10^{4}$ and was obtained from measurements ${ }^{10}$ carried out in modified Ostwald viscometer at $25.0^{\circ} \mathrm{C}$ using chlorobenzene as solvent. It has been previously purified by fractional precipitation using benzene as solvent and methanol as non-solvent.

\section{Apparatus and Measurements}

The vapor-pressure assembly used for the static vapor-pressure measurements of mixtures is described elsewhere. ${ }^{3}$ It consisted of a MKS Baratron pressure gauge, a vapor pressure cell and a vacuum manifold. Differential pressure up to $300 \mathrm{mmHg}(40.0$ $\mathrm{kPa}$ ) could be measured and variations of $0.003 \mathrm{mmHg}$ could be detected. The vapor pressure cell was immersed in a constant temperature bath in which its maximum fluctuation was $\pm 0.01^{\circ} \mathrm{C}$.

The vacuum manifold was constantly kept at about $70^{\circ} \mathrm{C}$ to prevent any condensation of vapor, and the whole assembly was thoroughly degassed over many hours prior to any series of measurements. For the liquid mixtures procedure, the two liquid components were inserted into the cell by successive distillations from two different ampoules. The amounts of the components forming the mixture were determined by weighing the ampoules before 
and after the distillations. Attainment of vapor-liquid equilibrium in the cell was assumed by a stirring magnetic bar, coupled with an external rotor driven by compressed air. Regarding the experiments carried out on polymer mixtures, a detachable pressure cell was used in the apparatus, where about $1.5 \mathrm{~g}$ of previously dessicated PDOL was inserted and slowly degassed through the "bellows" stopcocks. A desired amount of the corresponding liquid component was thereafter distilled into the cell to form the polymer solution which was constantly stirred during the period of measurement.

In order to compute the volume fractions used in this work, the molar volumes of the liquid components are needed. They were determined using an Anton Paar DMA-55 digital densimeter with an estimated accuracy of $1 \times 10^{-2} \mathrm{~kg} \mathrm{~m}^{-3}$. The operation of the densimeter is based on measuring the period of oscillation of a vibrating U-shaped tube filled with liquid substance. The densimeter had prealably been calibrated using purified air and redistilled water as reference samples. The results of molar volumes are found in Table 5 for DOL and BEN. On the other hand, the volume fraction of polymer in the polymer mixtures was converted from its mass fraction using the density of the corresponding liquid and that of semicrystalline PDOL found experimentally ${ }^{9}$ as $1.305 \mathrm{~g} \mathrm{ml}^{-1}$.

\section{RESULTS AND DISCUSSION}

\section{Monomer/Solvent Mixture: $\chi_{\mathrm{ms}}$ Parameter}

According to the original Flory-Huggins theory, ${ }^{11}$ the interaction parameter is computed using the enthalpy of mixing, $\Delta H_{\mathrm{m}}$. However, in order to take into account of the entropic contribution into the whole interaction pattern, it should be deduced from the excess free-energy of mixing, $\Delta G^{\mathrm{E}}$, through:

$$
\chi_{\mathrm{ms}}=\frac{\Delta G^{\mathrm{E}}}{R T x_{\mathrm{m}} \phi_{\mathrm{s}}}
$$

where $x_{\mathrm{m}}$ is the mole fraction of the monomer and $\phi_{\mathrm{s}}$ is the volume fraction of the solvent calculated by:

$$
\phi_{\mathrm{s}}=\frac{\left(1-x_{\mathrm{m}}\right) \bar{V}_{\mathrm{s}}}{x_{\mathrm{m}} \bar{V}_{\mathrm{m}}+\left(1-x_{\mathrm{m}}\right) \bar{V}_{\mathrm{s}}}
$$

Before applying equation 3 to the computation of $\chi_{\mathrm{ms}}, \Delta G^{\mathrm{E}}$ has to be obtained beforehand from experimental data of vapor pressure of the mixture. This has been achieved by means of an iterative method $^{3}$ based on the Wilson's equation ${ }^{12}$ with only two adjustable parameters. This equation usually yields an excellent fitting of the pressure data for the binary liquid mixtures where the intermolecular interactions are not excessively strong, such as in the present system. Moreover, it is the simplest expression which can be used among many available empirical ones where three or more adjustable parameters are required. Table I shows the vapor pressure data, P, for the DOL/BEN mixture for temperatures ranging from 20 to $40^{\circ} \mathrm{C}$, together with the values of the Wilson's constants, $A_{12}$

Table I. Vapour pressure, $P$, of the mixtures:

1,3-dioxolan $(\mathrm{m})+$ benzene $(\mathrm{s})$ at various temperatures

\begin{tabular}{cccccc}
\hline \multicolumn{6}{c}{$P / \mathrm{kPa}$} \\
\hline$x_{\mathrm{m}}$ & $20^{\circ} \mathrm{C}$ & $25^{\circ} \mathrm{C}$ & $30^{\circ} \mathrm{C}$ & $35^{\circ} \mathrm{C}$ & $40^{\circ} \mathrm{C}$ \\
\hline 0.0 & 9.999 & 12.679 & 15.895 & 19.698 & 24.325 \\
0.1150 & 10.280 & 13.062 & 16.390 & 20.331 & 25.087 \\
0.1690 & 10.373 & 13.170 & 16.583 & 20.580 & 25.398 \\
0.2586 & 10.546 & 13.396 & 16.865 & 20.938 & 25.838 \\
0.3191 & 10.610 & 13.489 & 17.003 & 21.122 & 26.086 \\
0.4580 & 10.772 & 13.708 & 17.281 & 21.504 & 26.568 \\
0.5734 & 10.839 & 13.796 & 17.408 & 21.689 & 26.819 \\
0.7082 & 10.817 & 13.799 & 17.423 & 21.753 & 26.923 \\
0.8553 & 10.733 & 13.704 & 17.329 & 21.674 & 26.876 \\
1.0 & 10.532 & 13.479 & 17.072 & 21.405 & 26.605 \\
\hline$A_{12}$ & 1.1096 & 0.0946 & 1.0780 & 1.0758 & 1.1092 \\
\hline$A_{21}$ & 0.7190 & 0.7333 & 0.7505 & 0.7559 & 0.8093 \\
\hline $\begin{array}{c}\text { Std } \\
\text { deviation }\end{array}$ & 0.0020 & 0.0015 & 0.0015 & 0.0013 & 0.0010 \\
\hline
\end{tabular}


Table II. $\chi_{\mathrm{ms}}$ parameter for the mixtures: 1,3-dioxolan $(\mathrm{m})+$ benzene $(\mathrm{s})$ at various temperatures

\begin{tabular}{ccccccc}
\hline \multicolumn{7}{c}{$\chi_{\mathrm{ms}} \pm 0.002$} \\
\hline$x_{\mathrm{m}}$ & $\phi_{\mathrm{s}}$ & $20^{\circ} \mathrm{C}$ & $25^{\circ} \mathrm{C}$ & $30^{\circ} \mathrm{C}$ & $35^{\circ} \mathrm{C}$ & $40^{\circ} \mathrm{C}$ \\
\hline 0.1 & 0.920 & 0.177 & 0.176 & 0.173 & 0.170 & 0.169 \\
0.2 & 0.836 & 0.176 & 0.175 & 0.173 & 0.169 & 0.168 \\
0.3 & 0.748 & 0.176 & 0.174 & 0.172 & 0.168 & 0.166 \\
0.4 & 0.657 & 0.175 & 0.174 & 0.171 & 0.167 & 0.164 \\
0.5 & 0.560 & 0.175 & 0.173 & 0.170 & 0.166 & 0.162 \\
0.6 & 0.460 & 0.175 & 0.172 & 0.169 & 0.165 & 0.159 \\
0.7 & 0.354 & 0.174 & 0.171 & 0.168 & 0.164 & 0.158 \\
0.8 & 0.242 & 0.174 & 0.170 & 0.167 & 0.163 & 0.157 \\
0.9 & 0.124 & 0.173 & 0.170 & 0.166 & 0.162 & 0.157 \\
\hline
\end{tabular}

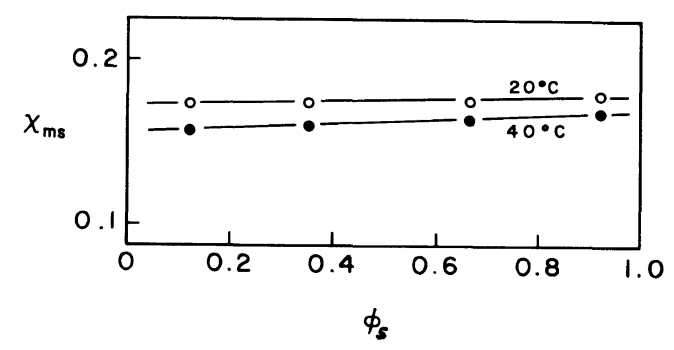

Figure 1. Variation of $\chi_{\mathrm{ms}}$ parameter with volume fraction of benzene for 1,3-dioxolan/benzene mixture.

and $A_{21}$, obtained after the iterative calculations. These constants allow for the evaluation of $\Delta G^{\mathrm{E}}$ for any concentration, and hence, to the computation of the $\chi_{\mathrm{ms}}$ parameter shown in Table II and Figure 1. For any given value of $x_{\mathrm{m}}, \phi_{\mathrm{s}}$ calculated from eq 4 was found to vary with temperature. However, this variation is very small (a variation of $2 \times 10^{-4}$ for the 20 to $40^{\circ} \mathrm{C}$ temperature range), so only average values of $\phi_{\mathrm{s}}$ are represented in Table II and Figure 1.

From the positive values of $\chi_{\mathrm{ms}}$, it appears that the monomer molecules generate only moderate interactions with the solvent. Strong attractive forces between the oxygens of DOL and the $\pi$ electrons of the aromatic ring are expected, but they are likely not strong enough to prevail over the powerful intermolecular forces found in the pure liquids, specifically among the DOL species. The latter behaves nearly like a multicyclic liquid due to its asymetric five-membered ring shape formed by three $-\mathrm{CH}_{2}-$ groups inserted by two oxygen atoms. The relatively small values of its molar volume, Table $\mathrm{V}$, may lend support to this finding. The same kind of interactions has also been observed in the mixtures of DOL with $p$-xylene,${ }^{13}$ chlorobenzene,${ }^{14}$ or methylcyclohexane ${ }^{15} \cdots$ where the $\Delta G^{\mathrm{E}}$ values were all positive. As the temperature increases, the vigorous molecular thermal agitation may gradually reduce the intermolecular forces between like molecules in favour of the intermolecular attractions between alike molecules. This effect can be observed through the decreasing values of $\chi_{\mathrm{ms}}$ with increasing temperature (Figure 1). In contrast with most polymer mixtures where the $\chi$ parameter varies largely with concentration, the $\chi_{\mathrm{ms}}$ parameter is virtually constant over the entire concentration range, making the use of this parameter in eq 1 and 2 most convenient. This constancy is usually observed in the mixtures of two nonassociated liquids where the interactions of the Van der Waals types are predominant, and, their molecular sizes are not much different. The above conditions make the lattice model of the Flory-Huggins theory most suitable to be applied to this type of binary liquid mixtures.

\section{Polymer/Liquid Mixtures: $\chi_{\mathrm{sp}}$ and $\chi_{\mathrm{mp}} \mathrm{Pa}$ - rameters}

The interaction parameters for BEN/PDOL and DOL/PDOL mixtures are computed directly from experimental data of vapor pressure, $P$, for each respective system, using:

$$
\ln a=\ln \left(1-\phi_{\mathrm{p}}\right)+\phi_{\mathrm{p}}+\chi \phi_{\mathrm{p}}{ }^{2}
$$

where $\phi_{\mathrm{p}}$ is the polymer volume fraction in the corresponding mixture and $\mathrm{a}$ is the activity of either BEN or DOL, which is calculated from $P / P^{0}$ after having been corrected for the nonideality of the vapor phase. ${ }^{4}$

Values of $P$, a and $x_{\mathrm{sp}}$ parameter at 30 and 
Table III. $\chi_{\mathrm{sp}}$ for the mixtures: benzene (s)/ poly(1,3-dioxolan) (p)

\begin{tabular}{ccccc}
\hline$t / 0^{\circ} \mathrm{C}$ & $\phi_{\mathrm{p}}$ & $P / \mathrm{kPa}$ & $a$ & $\chi_{\mathrm{sp}}$ \\
\hline \multirow{2}{*}{30} & 0.252 & 15.596 & 0.9812 & 0.305 \\
& 0.181 & 15.756 & 0.9913 & 0.302 \\
& 0.108 & 15.839 & 0.9965 & 0.240 \\
& 0.098 & 15.847 & 0.9970 & 0.218 \\
40 & 0.251 & 23.848 & 0.9804 & 0.290 \\
& 0.180 & 24.101 & 0.9908 & 0.285 \\
& 0.155 & 24.145 & 0.9926 & 0.248 \\
& 0.107 & 24.237 & 0.9964 & 0.225 \\
& 0.098 & 24.247 & 0.9968 & 0.210 \\
\hline
\end{tabular}

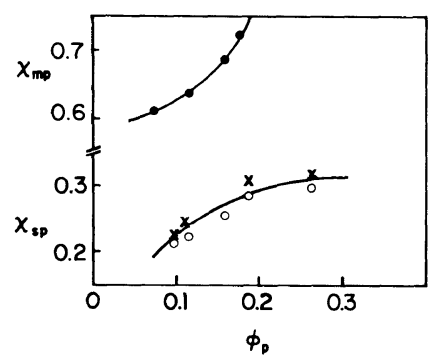

Figure 2. Variation of $\chi_{\mathrm{sp}}$ and $\chi_{\mathrm{mp}}$ parameters with volume fraction of polymer. $\times, \bigcirc$, for benzene/ poly(1,3-dioxolan) mixture at $30,40^{\circ} \mathrm{C}$; - for 1,3 dioxolan/poly(1,3-dioxolan) mixture at $40^{\circ} \mathrm{C}$.

$40^{\circ} \mathrm{C}$ are presented in Table III and Figure 2. The relatively small values of $\chi_{\text {sp }}$ indicate that rather strong attractions can be established between BEN molecules and PDOL chains likely owing to interactions between oxygen atoms of linear polymer chains and hydrogen atoms of aromatic rings which readily surround the former. This seems to be in contrast with the case of cyclic DOL molecules where intermolecular interactions with BEN molecules are hindered by strong intermolecular forces within each liquid component.

Measurements were performed at $40^{\circ} \mathrm{C}$ on the DOL/PDOL mixture but solubility appeared to be poor for this system and high values of $\chi_{m p}$ (Table IV and Figure 2) are obtained. The limit of solubility of the polymer is for $\phi_{\mathrm{p}}$ of about 0.20 . This can be understood by the fact that no strong interactions can be
Table IV. $\chi_{\mathrm{mp}}$ for the mixture: 1,3-dioxolan (m)/ poly(1,3-dioxolan) (p)

\begin{tabular}{ccccc}
\hline$t / 0^{\circ} \mathrm{C}$ & $\phi_{\mathbf{p}}$ & $P / \mathrm{kPa}$ & $a$ & $\chi_{\mathrm{mp}}$ \\
\hline \multirow{2}{*}{40} & 0.185 & 26.743 & 1.0052 & 0.723 \\
& 0.162 & 26.685 & 1.0030 & 0.677 \\
& 0.115 & 26.637 & 1.0012 & 0.635 \\
& 0.078 & 26.618 & 1.0005 & 0.610 \\
\hline
\end{tabular}

created between the linear structure of PDOL and the cyclic configuration of DOL molecules in order to overcome the powerful intermolecular forces within the latter. This poorly compatible monomer/polymer system is completely in contrast with a previously reported cyclic monomer/linear polymer, the tetrahydrofuran/poly(tetrahydrofuran) system $^{16}$, where interactions were found to be surprisingly strong. The above noteworthy contrast may be explained by the fact that DOL molecules contain two oxygen atoms for three $-\mathrm{CH}_{2}-$ groups while tetrahydrofuran has just one oxygen atom for four $-\mathrm{CH}_{2}-$ groups. Such a high population density of oxygen in DOL/PDOL system, as previously mentioned tends to favor attractive interactions between like molecules at the expense of those between unlike molecules, saying DOL and PDOL ones. The large increase of $\chi_{\mathrm{mp}}$ parameter with concentration is a common behavior of "poor" solvent/polymer mixtures.

\section{Computation of $\beta$ and $\Delta G_{\mathrm{lc}}$}

The interaction parameters obtained above, $\chi_{\mathrm{ms}}, \chi_{\mathrm{sp}}$, and $\chi_{\mathrm{mp}}$, can now be used together with the available data ${ }^{10}$ for the equilibrium polymerization of DOL in BEN to calculate the solvent effect term, $\beta$, and the free-energy change upon the conversion of DOL into PDOL, $\Delta G_{\mathrm{lc}}$, through eq 2 and 1 . In carrying out these computations, the values of each parameter had to be extrapolated from the corresponding figures, Figures 1 and 2, in order to take into account their concentration dependence. The values of $\Delta G_{\mathrm{Ic}}$ are then compared to the ones obtained from the method ${ }^{3}$ 
Table V. $\beta$ and $\Delta G_{\mathrm{lc} / R T}$ for the polymerization of 1,3-dioxolan in benzene, with $\chi_{\mathrm{mp}}=0.50$

\begin{tabular}{cccccccc}
\hline \multicolumn{1}{c}{$\frac{\bar{V}_{\mathrm{m}}}{{ }^{\circ} \mathrm{C}}$} & $\frac{\bar{V}_{\mathrm{s}}}{\mathrm{ml} \mathrm{mol}^{-1}}$ & $\frac{\mathrm{ml} \mathrm{mol}^{-1}}{n n n n y y y}$ & & & $\frac{\Delta G_{\mathrm{lc}}}{R T}$ & $\frac{\Delta G_{\mathrm{lc}}{ }^{\mathrm{a}}}{R T}$ \\
\hline 20 & 69.70 & 88.87 & -0.108 & -1.26 & -1.34 \\
40 & 71.35 & 91.12 & -0.097 & -0.97 & -0.74 \\
50 & 72.25 & 92.30 & -0.085 & -0.75 & -0.74 \\
60 & 73.20 & 93.55 & -0.070 & -0.55 & -0.54 \\
\hline
\end{tabular}

${ }^{a}$ From ref 8 .

proposed for the cases when the interaction parameters are not available. A fixed value of 0.50 has been used for $\chi_{\mathrm{mp}}$ parameter in all above calculations, provided that it has only a minor contribution into eq 1 due to usually small values of $\left(\phi_{\mathrm{p}}-\phi_{\mathrm{m}}\right)$ term encountered in polymerization systems carried out in solution. According to the results presented in Table $\mathrm{V}$, both sets of values of $\Delta G_{\mathrm{Ic}} / R T$ are in good agreement except for the ones corresponding to $40^{\circ} \mathrm{C}$. In that case, experimental errors on polymerization data greatly affect the method ${ }^{3}$ and may lead to erroneous results. In a recent work, ${ }^{4}$ it has been argued that, on the thermodynamic standpoint, a high extent of conversion of monomer into polymer could be achieved if the monomer/solvent interactions were as weak as possible while those between solvent and polymer should be as strong as possible, yielding $\beta$ values as high as possible. Such criteria seem to be fairly fulfilled with $\mathrm{BEN}$ as a solvent for the polymerization of DOL.

The key idea of the present method for computing $\Delta G_{\mathrm{lc}}$ and $\beta$ using three binary interaction parameters is to assume the "living" equilibrium polymerization system to behave thermodynamically like a nonreactive ternary polymer solution (one polymer with two solvents) where the living polymer chains are considered as terminated polymer. This assumption leads to neglect any kind of interaction arisen from the ionic chain ends as well from the counter-ions. This condition could be fulfilled if: (i) The polymerization systems dealt with are to produce polymers of high molecular-weight so that the molar concentrations of ionic active ends are small compared to those of monomer and solvent; (ii) The solvent used is a low polar liquid with a small dielectric constant so that a "living" polymer chain-counterion pair could be regarded as still being in the undissociated state, since the dissociation ability of electrolytes increases along with dielectric strength; consequently, the interionic interactions between neighbouring "living" chains would become minimized.

\section{CONCLUSION}

The thermodynamic behavior of a living equilibrium polymerization system in solution can be considered as that of a nonreactive ternary polymer solution. It can be adequately represented by only three binary interaction parameters: $\chi_{\mathrm{ms}}, \chi_{\mathrm{sp}}, \chi_{\mathrm{mp}}$, so long as their dependence on concentration is taken into account. That condition is satisfied through the use of their real value at a given concentration obtained from interpolations on their respective graph of $\chi$ plotted against the concentration. However, in the cases where some specific interactions like hydrogen bonding, for instance, are present in the ternary system, it can be expected that the above three binary parameters would not be accurately representative so that an additional ternary interaction parameter should be involved in such a manner that only the $\chi_{\mathrm{ms}}$ parameter will be modified ${ }^{16}$ while the other two binary parameters remain the same. In future work, a method will be developed to deduce the ternary parameter from experimental vaporpressure data of ternary polymer mixtures.

\section{REFERENCES}

1. H. Sawada, "Thermodynamics of Polymerization," Mercel Dekker, New York, N. Y., 1976, Chapter III.

2. J. Léonard, Macromolecules, 2, 661 (1969). 
3. V. T. Bui and J. Léonard, J. Chem. Soc., Faraday Trans. 1, 81, 1745 (1985).

4. J. Léonard and V. T. Bui, Polymer, 28, 1041 (1987).

5. K. J. Ivin and J. Léonard, Eur. Polym. J., 6, 331 (1970).

6. J. Léonard and S. L. Malhotra, J. Polym. Sci., A-1, 9, 1983 (1971).

7. R. E. Cunningham, Polymer, 19, 729 (1978).

8. L. I. Kuzub, M. A. Markevich, A. A. Berlin, and N. S. Yenikolopyan, Vysokomol. Soedin, 10, 2007 (1968).

9. R. Binet, Ph. D. thesis, Laval University, Québec, 1974.

10. J. Brandrup and . E. H. Immergut, "Polymer
Handbook," Wiley \& Sons, New York, N. Y., 1975, Chapter IV.

11. P. J. Flory, "Principle of Polymer Chemistry," Cornell University Press, Ithaca, N. Y., 1953, Chapter XII.

12. G. M. Wilson, J. Am. Chem. Soc., 86, 127 (1964).

13. C. Castellari, R. Francesconi, and F. Comelli, J. Chem. Eng. Data, 27, 156 (1982).

14. R. Francesconi and F. Comelli, J. Chem. Eng. Data, 30, 352 (1985).

15. C. Castellari and R. Francesconi, and F. Comelli, Can. J. Chem. Eng., 66, 131 (1986).

16. V. T. Bui and J. Léonard, J. Chem. Soc., Faraday Trans. 1, 82, 899 (1986). 